\title{
GESTURENAIL:WIRELESS HAND GESTURE SYSTEM
}

\author{
Emi Tamaki1 and Ken Iwasaki2 \\ 1Waseda University, Tokorozawa, JAPAN \\ 2H2L Inc., Tokyo, JAPAN
}

\begin{abstract}
The hand gesture input systems will be adopted for use in many applications anytime and anywhere. In this research, a stating notification when and where a user can start using hand gestures for input are focused. However existing devices and systems could be used only in certain situations because of safety issues and the limitations of the devices.

In this paper, a Wireless Nail Gesture System is suggestted and we developed a proof-of-concept model of the Wireless Nail Gesture System that can be installed on artificial nails using an LED and a coil. The LED light notifies whether the finger is within a RF(Radio Frequency) gesture space that is the magnetic field generated by a transmission antenna. The RF supplies the power and the signal. The greatest benefit of this system is that users can wear the device anytime and anywhere. In addition, by measuring the impedance of the transmission antenna, the system distinguishes three states: (1) outside the gesture space, (2) ON gesturewithin the gesture space and (3) OFF gesture within the gesture space. For example, to indicate the ON gesture, the user covers the LED on the fingernail with another finger.

A usability study of thesuggested system determined how the notification system affected the users' success rate and speed at searching for a hand gesture space. If the system does not notify the user to start, the task success rate drops to $85 \%$. If the system notifies the user to start, the task success rate is $100 \%$ in all sessions. The experiment also revealed that the start notification reduces the task time by $0.7 \mathrm{~s}$ from 22.1 s.
\end{abstract}

\section{KEYWORDS}

Hand gesture, RF(radio frequency), fingernail, wearable,human-computer interaction, user interface, ubiquitous computing, design

\section{INTRODUCTION}

Many hand gesture input systems have been suggested in the fields of HCI (Human-Computer Interaction) and Ubiquitous Computing to be adopted for use in many applications anytime and anywhere. However, users need to be notified about when and where hand gestures are accepted as input. In this paper, we focus on this notification.

The feedback devices for the hand gesture input are categorized into three types: touch panel type,room-mounted device type and wearable type:

DOI : 10.5121/ijcsea.2013.3503 
Touch panel type The touch-panel devices [1-6] generate audio, visual, and tactile feedbacks via the touch panel when it recognizes the user's hand gestures on the panel. These devices can be used without consistent pointing, because the input sensors and feedback actuators are installed in the same place. Users can start using hand gestures for input only after user lays a hand on the panel. This type of device limits the hand gesture variations, because it requires touching the panel.

Room-mounted device type The room-mounted devices give the user audio and visual feedbacks using speakers and projectors [7-9], which notify the user when and where hand gestures can be used as input. However, the sound could be an unwelcomed noise, and the notification images could be projected only within a dark space. As an exception, a room-mounted type device can also include haptic feedback using airborne ultrasound [10]. Such device gives the user a tactile feeling anywhere and can be used as feedback to notify the user when and where to use hand gestures as input. However, the device generates a maximum of 0.8 gram-forces [10], which is too weak to use for notifications.

Wearable type The wearable devices vary according to the kind of feedback they provide. In this section, we discuss feedback devices that can be used to notify the user to start using hand gestures for input.

Head-mounted display (HMD) devices, such as Google Glass [11] and AiRScouter [12], are used for visual feedback and have become small and lightweight in the form of eyeglasses. However, these devices must be taken off for the user's safety in some situations, such as in the bedroom or the bathroom.

Other devices use a small projector as a wearable feedback device. Sixth Sense [13] and Brainy Hand $[14,15]$ both have a battery, a small camera to detect the user's hand gestures, and a small projector for visual feedback. These devices are worn on the user's head or neck to avoid hindering the user's gestures, and the visual feedback is projected on the user's hand.

Users can enter input through hand gestures anywhere; however, these systems need a boot-up command or a switch to turn the system on and off in order to avoid confusing the user's daily hand gestures with hand gesture commands. For mobility, these systems also need a battery, which the user must carry. Therefore, the two systems are unsuitable for quick hand-gesture input in daily use.

For the audio feedback, ordinary Bluetooth earphones [16] can be used, but they must also be taken off in some cases for the same reasons as the HMD devices.

The Master Slave[17] and TELESAR[18] devices provide tactile feedback through specialized gloves to allow the user to control a robot hand. The tactile feeling is synchronized with the robot hand's movements. These devices cannot be used on a daily basis, because they cover the user's hands and inhibit hand motions. PossessedHand [19] is a device that controls the user's finger joint movements by using non-invasive electric muscle stimulation (EMS) around the user's arm. In order to maintain the health of the skin and muscles, PossessedHand is allowed to be used only within a limited period of time. 
Exception Ubi-Finger [20, 21] is designed to use finger gestures as input in a ubiquitous environment and provides feedback using only the behavior of the controlled devices. Ubi-Finger allows a user to select a target device by pointing a finger; however, the sensors cover the user's finger, and the electric leads hinder the user's normal movements.

Various feedbacks could be used as the start notification. However, to be able to use the device anytime and anywhere, the following criteria are required:

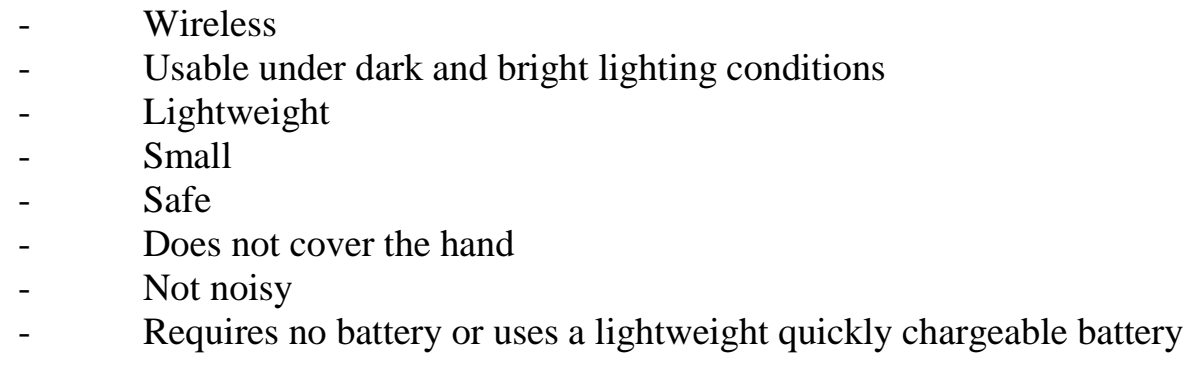

Our goal is to develop a start notification system that meets the above criteria. In this paper, we suggest a Wireless Nail Gesture System that is installed on the user's fingernail. The power and the signal are supplied by the radio frequency. With this system, the user can wear and use the start-notification device anytime and anywhere.

\section{SYSTEM CONFIGURATION}

The Wireless Nail Gesture System can be used both as an input system for the computer and as a notification system for the user. The device, which consists of a coil and an LED, is mounted on the user's fingernail. The user can activate the system by first covering the LED on the fingernail using another finger [Figure 1]. When the user makes this ON gesture, the resistance of the device changes.

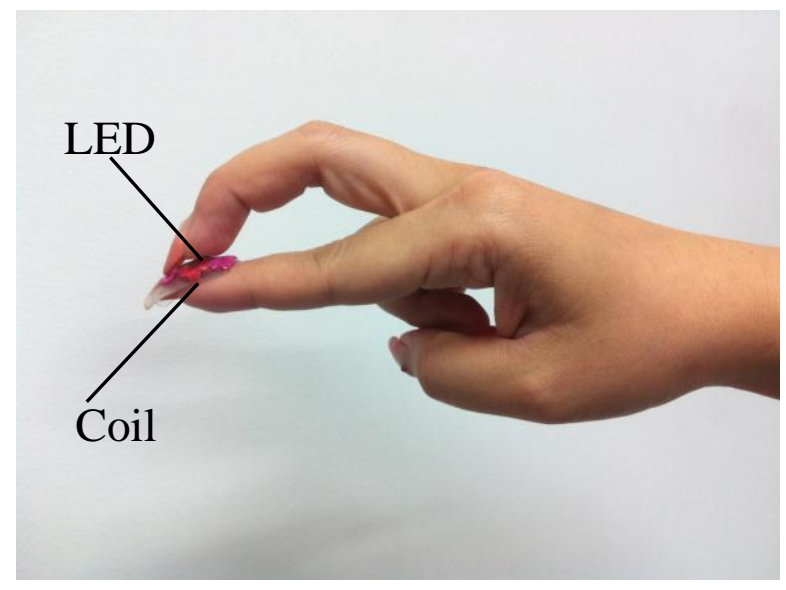

Figure 1. ON gesture for Wireless Nail Gesture System. 


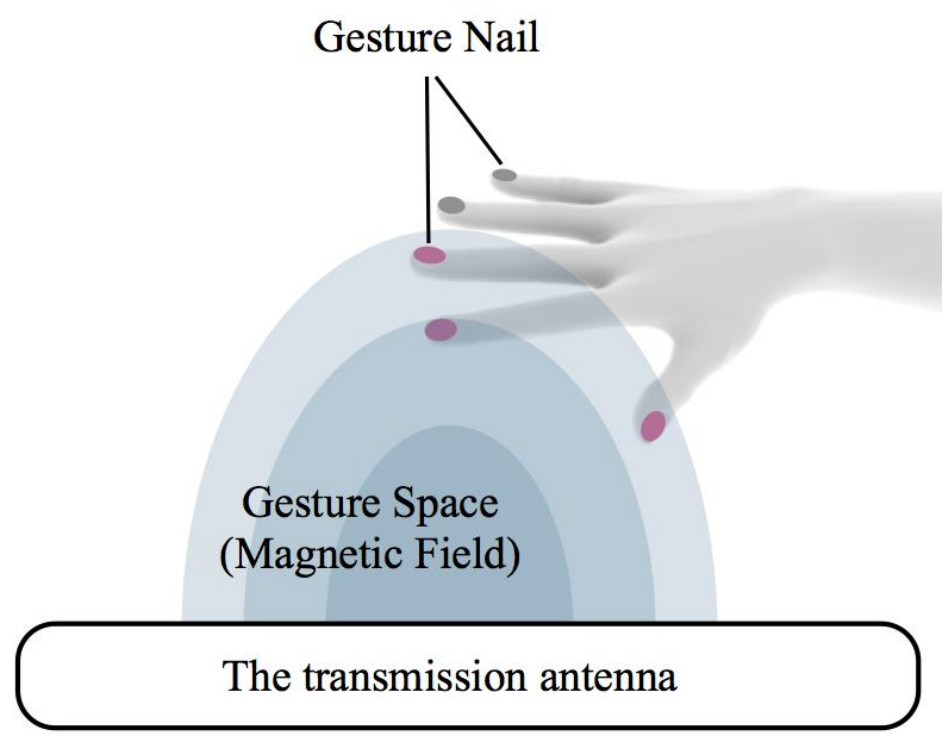

Figure 2. Gesture space. Three fingers (index, middle, and thumb) are within the gesture space, whereas two fingers (ring and little) are outside the gesture space.

The device is electromagnetically coupled to the near field communication (NFC) tag reader; therefore, the resistance change of the LED results in an impedance change in the NFC tag reader, and the computer can determine whether the user made an $\mathrm{ON}$ gesture or not.

When the user places his/her hand in the gesture space, the LED turns on, using power supplied by the NFC, to notify the user when and where to make hand gestures. The LED functions as both the sensor for gesture detection and the notification device for the user.

The system uses radio frequency (RF) to obtain power wirelessly; therefore, it does not need a heavy battery, and it does not interfere with the user's daily motions.

The gesture space of the Wireless Nail Gesture System is a magnetic field generated by the transmission antenna of the NFC tag reader. Figure 2 shows the image of the gesture space of the system, where the middle finger, the index finger, and the thumb are within the gesture space, whereas the ring finger and the little finger are outside the gesture space. Because we cannot see the magnetic field, the system runs the LED on to indicate that three fingers are within the gesture space.

As a proof of concept, we built a prototype system using a surface-mounted type LED (Siemens LST676-R, 20mA) and a coil. For the NFC tag reader, we used a SONY RC-S320, which emits a $13.56 \mathrm{MHz}$ radio frequency. Using a $0.2 \mathrm{~mm}$ diameter tinned wire, we created a coil with 16 loops and a diameter of $15 \mathrm{~mm}$ to make it fit an artificial nail [Figure 3]. 

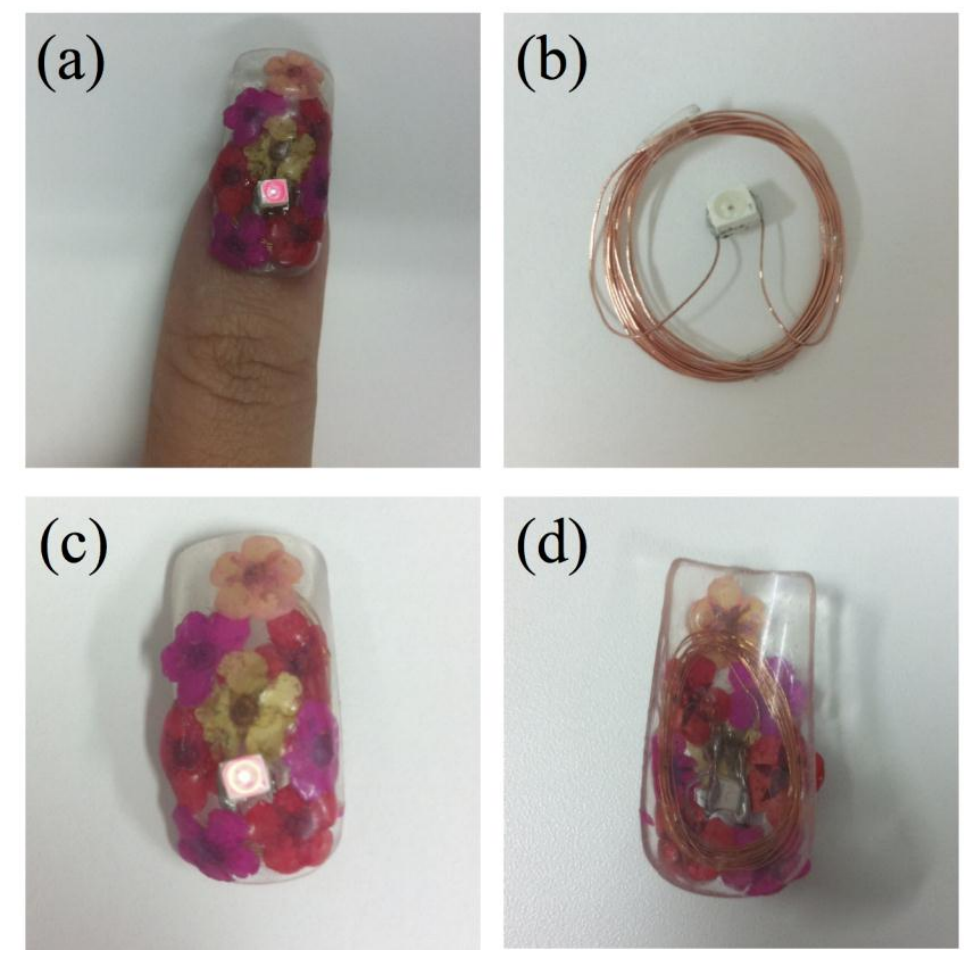

Figure 3. Prototype of the Wireless Nail Gesture System. (a) Prototype on the index finger. (b) Circuit (LED and coil). (c) Prototype mounted on the artificial nail. (d) Bottom view of (c).

The size can be reduced by changing the diameter of the coil or the number of turns. The inductance of the coil was set to $5.9 \mu \mathrm{H}$ for electromagnetic coupling.

As a preliminary experiment, we studied how the Wireless Nail Gesture System affects the magnetic field generated by the transmission antenna. Using a Wheatstone bridge circuit and operational amplifier, we measured the impedance of the transmission antenna on the NFC tag reader. Figure 4 shows the results under three conditions: (1) the hand is outside the gesture space, (2) the ON gesture within the gesture space, and (3) the OFF gesture within the gesture space. The ON gesture is defined as covering the LED on the fingernail with another finger [Figure 1] while inside the gesture space. The OFF gesture is defined as uncovering the LED while inside the gesture space.

The amplified impedance clearly drops when the user places the hand in the gesture area (the magnetic field generated by the transmission antenna), because the antenna of the NFC tag reader is coupled to the device on the user's fingernail.

When the user covers the LED (ON gesture), the resistance of the LED becomes slightly higher than the OFF gesture. As a result, the impedance of the antenna in the NFC tag reader becomes lower. 


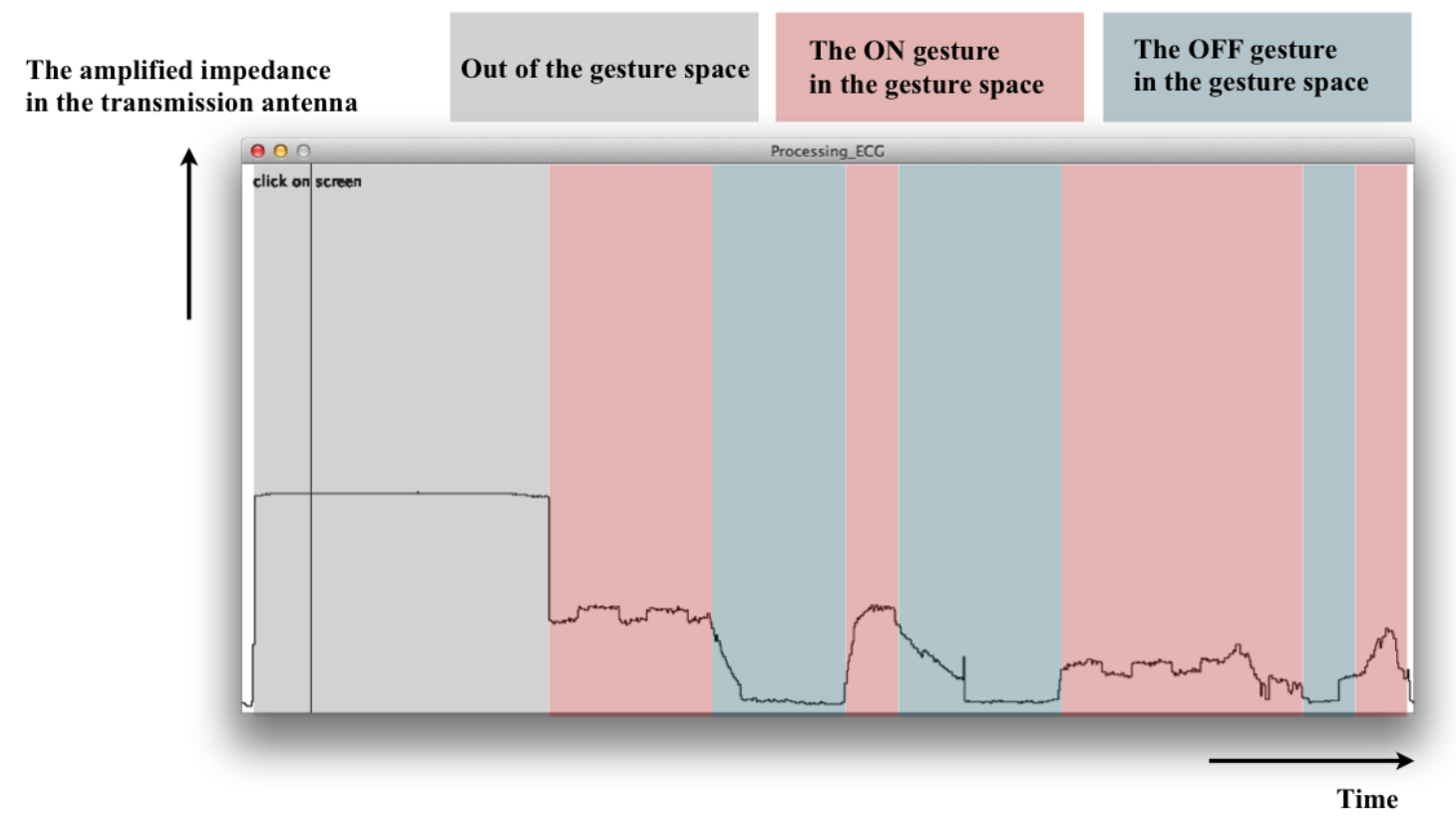

Figure 4. Example showing changes in impedance signal caused by the gestures. (1) User's hand is outside the gesture space (gray). (2) The gesture is ON within the gesture space (pink). (3) The gesture is OFF within the gesture space (blue).

Thus, the system distinguishes the ON and OFF gestures. When the gesture is ON, the impedance shows a flat signal, which is assumed to be the result of the communication signal from the NFC tag reader.

In this simple experiment, we confirmed that the system can distinguish three states: (1) The user's hand is outside the gesture space. (2) The user makes the ON gesture within the gesture space. (3) The user makes the OFF gesture within the gesture space.

\section{EXPERIMENTS}

We hid the NFC tag reader under an opaque acryl box so that the subjects cannot determine its location. First, the subjects were told to place their hand in a fixed position. Then, they were told to find the gesture space (above the NFC tag reader) and make the ON gesture in that space. Afterwards, they were instructed to place their hand back in the previous fixed position. They are told to repeat these steps 10 times as fast as possible. We asked the subject to do this session 5 times. That is, we measured 50 gesture trials under one condition. We used two artificial nails; one with LED feedback and another without LED feedback. With these additional conditions, each subject performed 100 trials. We measured the differences in efficiency and speed.

\subsection{Efficiency}

The task success rate is shown in Figure 5. With LED feedback, the task success rate was $100 \%$ over all sessions. Without LED feedback, the task success rate dropped to $85 \%$, because the subjects were not sure if they placed their hand within the gesture space without any LED feedback. Therefore, the notification from the LED is sufficient in finding the gesture space. 


\section{Task Success Rate}

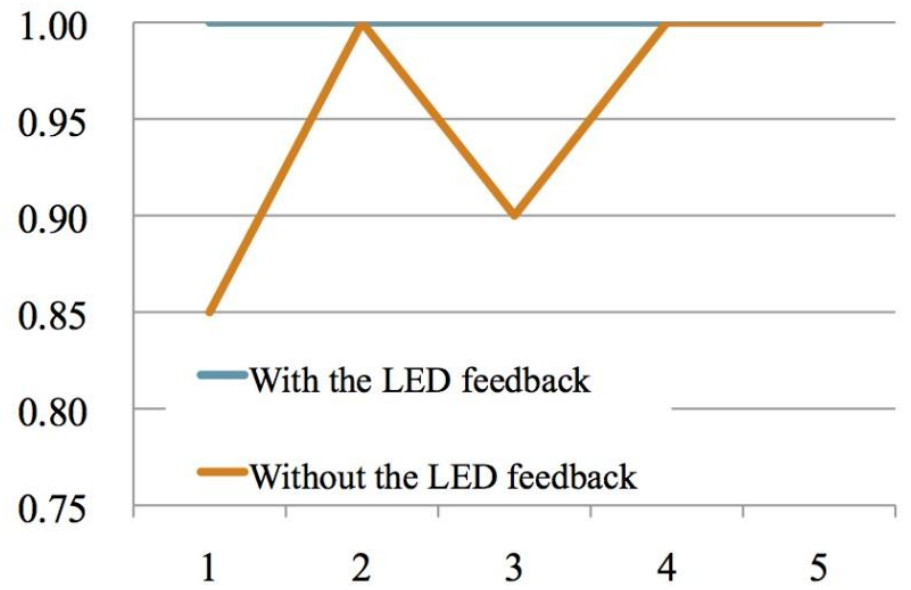

Figure 5. Efficiency measurements. With LED feedback, the gesture is $100 \%$ successful; without LED feedback, the recognition of the gesture is unpredictable.

\section{Time $(\mathrm{sec})$}

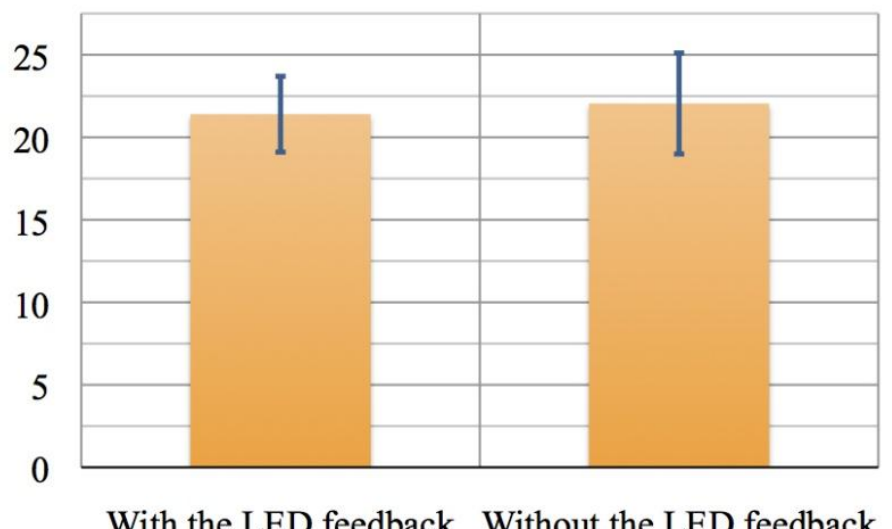

Figure 6. Speed measurements. The average speed differs only slightly under different conditions; however, the variance differs significantly.

\subsection{Speed}

The average task speed in the two conditions is shown in Figure 6. The average time was 21.4 seconds for trials with LED feedback and 22.1 seconds without LED feedback. The variance also shows a difference depending on the conditions: 2.29 with LED feedback, 3.05 without LED feedback.

With LED feedback, the user can find the gesture space more quickly within a relatively predictable time. Without LED feedback, the user is not sure if they placed the hand over the gesture space, which leads to self-doubt, resulting in longer time and higher variance. 


\section{DiSCUSSION}

Our Wireless Nail Gesture System can be applied to several types of applications.

\subsection{Application1: Controlling Home Electronics}

Our method can be used as a controller of home electronics, such as TVs, audio systems, and air conditioners. Unlike an ordinary remote controller, the Wireless Nail Gesture System can offer a seamless user experience in a daily situation, because it does not require a bulky battery or a complicated circuit. Moreover, it can be implemented as a waterproof device, so that it can be used even in the shower room or in the bathtub.

\subsection{Application2: Controlling Public Space Electronics}

Our Wireless Nail Gesture System can be manufactured at low cost, because it is comprised of only an LED and a coil; therefore, it can become a commodity device, like pens. Anybody wearing our device can control public-space electronics, like elevators or traffic lights. Instead of pressing a button to cross the road, users can simply cover their fingertip.

\subsection{Application3: Adding a "Click" Experience into Tablet Devices}

Tablet computers require a different user interface, and many applications use combinations of tapping and swiping gestures to indicate "click" or "drag-and-drop" commands. Our Wireless Nail Gesture System can tell whether the user makes an ON gesture or not on the tablet surface to enable mouse-like operations, like "click" or "drag and drop."

\section{CONCLuSiOnS}

In this paper, we suggested the Wireless Nail Gesture System, which can notify the user when and where hand gestures can be used as input anytime and anywhere. We developed a proof-ofconcept model of the suggested system, which uses an LED and a coil and can be installedon an artificial nail. The LED light notifies the user whether the finger is within theradio frequency (RF) gesture space of a transmission antenna. The power and the signal are supplied by the RF. The greatest benefit of this wearable system is that the user can use the start notification device anytime and anywhere. In addition, by measuring the impedance of the transmission antenna, the system distinguishes three states: (1) outside the gesture space, (2) ON gesture within the gesture space, and (3) OFF gesture within the gesture space. For example, to indicate the ON gesture, the user covers the LED on the fingernail with another finger.

A usability study of oursystem determined how the notification system affected the users' success rate and speedat finding hand-gesture space. Without notifying the user to start, the task success rate dropped to $85 \%$. With the start notification, the task success rate was $100 \%$ in all sessions. The experiment revealed that the starting notification reduced the task time by $0.7 \mathrm{~s}$ from $22.1 \mathrm{~s}$. Finally, three future applications were discussed: a home electronics control application, a public space electronics control application, and the "Click" gesture on the surface of tablet devices that can identify whether the user made an ON gesture or not. 
International Journal of Computer Science, Engineering and Applications (IJCSEA) Vol.3, No.5, October 2013

\section{ACKNOWLEDGEMENTS}

The authors would like to thank an anonymous reviewer and an editor for their feedback and great suggestions. We also thank all subjects for their participation in the experiments and our friends for the discussion.

\section{REFERENCES}

[1] J. Rekimoto(2002)"SmartSkin: an infrastructure for freehand manipulation on interactive surfaces", ACM, Proceedings of the SIGCHI Conference on Human Factors in Computing Systems, No. 8, pp. 113-120.

[2] Han Y. Jefferson(2005)“Low-cost multi-touch sensing through frustrated total internal reflection", ACM, Proceedings of the 18th annual ACM symposium on User interface software and technology, pp.115-118.

[3] iPad(2010), Apple.Inc, http://www.apple.com/ipad/.

[4] Microsoft PixelSense and Microsoft Surface(2007), Microsoft Corporation, http://www.microsoft.com/en-us/pixelsense/.

[5] H. Kajimoto(2012) "Skeletouch: transparent electro-tactile display for mobile surfaces", ACM, SIGGRAPH Asia 2012 Emerging Technologies, pp. 21:1-21:3.

[6] M. Sato, I. Poupyrev, and C. Harrison(2012)“Touché: Enhancing Touch Interaction on Humans, Screens, Liquids, and Everyday Objects", ACM, Proceedings of the SIGCHI Conference on Human Factors in Computing Systems, No. 10, pp.483-492.

[7] G-Speak(2005), oblong industries, inc, http://www.oblong.com/g-speak/.

[8] Gallo, L. Placitelli, A.P. and Ciampi, M(2011) "Controller-free exploration of medical image data: Experiencing the Kinect”, IEEE, Computer-Based Medical Systems (CBMS), 2011 24th International Symposium on, pp. 1-6.

[9] R. Xao, C. Harrison and Scott E.Hudson(2013)“WorldKit: rapid and easy creation of ad-hoc interactive applications on everyday surfaces", ACM,Proceedings of the SIGCHI Conference on Human Factors in Computing Systems, pp. 879-888.

[10] T.Iwamoto, M.Tatezono and H. Shinoda(2008)"Non-Contact Method for Producing Tactile Sensation Using Airborne Ultrasound", Proc. EuroHaptics 2008, LNCS 5024, pp. 504-513.

[11] Google Glass(2012), Project Glass, Google.Inc., http://www.google.com/glass/start/.

[12] AiRScouter(2008), Brother Industries, Ltd., http://www.brother.co.jp/product/hmd/wd100ga/index.htm .

[13] P. Mistry, P. Maes and L. Chang,(2009)“WUW - Wear Ur World - A Wearable Gestural Interface", ACM, In the CHI '09 extended abstracts on Human factors in computing systems, No. 6, pp.41114116.

[14] E.Tamaki, T.Miyaki and J.Rekimoto(2009)"Brainy hand: an ear-worn hand gesture interaction device", ACM, In the CHI '09 extended Abstracts on Human Factors in Computing Systems, No. 6, pp.4255-4260.

[15] E.Tamaki, T.Miyaki and J.Rekimoto(2010)"BrainyHand: a wearable computing device without HMD and it's interaction techniques", Proceedings of the International Conference on Advanced Visual Interfaces, No. 2, pp.387-388.

[16] Bluetooth Wireless hadphone 903+ (2010), Plantronics.Inc.,http://www.plantronics.com/us/product/backbeat-903-plus .

[17] K. Sato, K. Minamizawa,N.Kawakami and S.Tachi(2007), MasterSlave in "Haptic Telexistance", ACM,Proceedings of the SIGCHI Conference on Human Factors in Computing Systems, SIGGRAPH2007 Emerging Technologies.

[18] C. L. Fernando, M. Furukawa, T. Kurogi,K. Hirota, S. Kamuro and K. Sato, K. Minamizawa and S. Tachi(2012) "TELESAR V: TELExistence surrogate anthropomorphic robot”, ACM, Proceedings of 
International Journal of Computer Science, Engineering and Applications (IJCSEA) Vol.3, No.5, October 2013

the SIGCHI Conference on Human Factors in Computing Systems, SIGGRAPH2012 Emerging Technologies, pp. 23:1.

[19] E.Tamaki, T.Miyaki and J.Rekimoto(2011) "PossessedHand: techniques for controlling human hands using electrical muscles stimuli", Proceedings of the SIGCHI Conference on Human Factors in Computing Systems, No. 10, pp.543-552.

[20] K. Tsukada and M. Yasumura(2001)“Ubi-Finger: Gesture Input Device for Mobile Use", Companion Proceedings of Ubicomp'2001, Technical Report: GIT-GVU-TR-01-7.

[21] K. Tsukada and M. Yasumura(2004)"Ubi-Finger: a Simple Gesture Input Device for Mobile and Ubiquitous Environment", journal of Asian Information, Science and Life(AISL),Vol.2, No.2, Nova Science Inc., pp. 111-120. 